3 Research Square

\title{
Renal Infarction Associated with Atrial Fibrillation Following ICD Implantation: A Case Report and Literature Review
}

\section{Yingshuo Huang}

Beijing Friendship Hospital,Capital Medical University

Ying Sun ( $\nabla$ yingsun15@163.com )

Capital Medical University Affiliated Beijing Friendship Hospital https://orcid.org/0000-0002-76679721

\section{Case Report}

Keywords: Atrial fibrillation, ICD implantation, Periprocedural anticoagulation, Renal infarction,

Posted Date: August 17th, 2021

DOI: https://doi.org/10.21203/rs.3.rs-775830/v1

License: (c) (i) This work is licensed under a Creative Commons Attribution 4.0 International License.

Read Full License 


\section{Abstract}

Background: Atrial fibrillation increases the risk of thromboembolism in the kidney. Renal infarction in atrial fibrillation receiving implantable cardioverter defibrillator (ICD) treatment is rarely seen.

Case Presentation: We reported a case of a 76-year-old woman who presented with palpitations and short of breath with ECG showing paroxysmal atrial fibrillation (AF) and long RR interval. The patient was diagnosed congestive heart failure with NYHA class III-IV and received an ICD implantation as the onset of ventricular tachycardia. The seventh after ICD implantation operation, she complained of right lower abdominal pain with fever and blood WBC increased. Right renal infarction was confirmed after abdominal enhancement computed tomography scan. Anticoagulation therapy was given immediately, the patient had an stable international normalized ratio (INR) and mild elevated serum creatinine levels during follow-up.

Conclusion: This case enlightened us some comprehension about the strategy for periprocedural anticoagulation and prevention of thromboembolism in the elderly with paroxysmal atrial fibrillation. Renal infarction and anticoagulation strategies for atrial fibrillation during perioperation were reviewed in this paper.

\section{Background}

Acute renal infarction is a relatively rare cause of acute abdominal pain, and its diagnosis is often overlooked or delayed. A national prospective observational cohort study reported a frequency of $0.007 \%$ of acute renal infarction in approximately 250,000 patients who complained of abdominal pain in the emergency department(ED) ${ }^{[1]}$. Atrial fibrillation $(\mathrm{AF})$ and renal artery injury are the major causes of renal infarction ${ }^{[2-4]}$, perioperative anticoagulation strategies remain important for the prevention of thromboembolic events, particularly inpatients with AF and a high risk of thromboembolism., There was some evidence-based series and guidelines for the indications and strategies of anticoagulation already, while the truly frequency of anticoagulation in AF population is less than expectation in real world

\section{Case Report}

A 76-year-old woman complained of dyspnea for one month with exacerbation forthree days attended the clinic. Her history included paroxysmal AF, hypertension, and coronary atherosclerotic heart disease. Seven years ago, shereceived a DDD pacemaker due to syncope, which was induced by the frequent onset of a long RR intervals (the maximum was $4510 \mathrm{~ms}$ ) by Holter examination. She maintained sinus rhythm before a sudden ventricular tachycardia (VT) attack and had no oral or injected anticoagulants in the past two years. She was a complaint of syncope at the emergency department, and ECG revealed paroxysmal ventricular tachycardia. After an electrical conversion in the emergency department, the ventricular tachycardia terminated and an atrial fibrillation initiated. She was then admitted to the cardiac care unit (CCU), Department of Cardiology, Beijing Friendship Hospital. Physical examination revealed 
that blood pressure was $106 / 59 \mathrm{mmHg}$ with a heart rate of 102 beats per minute and a pulse rate of 90 beats per minute. The temperature was $36.5^{\circ} \mathrm{C}$. There was slight rale in both basal lungs, and the heart rhythm was irregular with no cardiac murmur. There was mild edema in both lower extremities.

Laboratory analysis showed complete blood count (CBC), urinalysis, coagulation function, myocardial enzymes, and kidney function were normal. ECG showed AF rhythm with no evidence of abnormal ST-T change like acute myocardial infarction. Renal ultrasound showed that kidney size and renal blood flow signals were normal. The patient received implantable cardioverter defibrillator (ICD) implantation within 48 hours after VT attack. She received no anticoagulation therapy during the periprocedure given the age, bleeding risk of pocket hematoma, and duration of AF less than 48 hours. Seven days after theimplantation, the patient presented abdominal pain with diarrhea, vomit, and fever $\left(T=38.7^{\circ} \mathrm{C}\right)$, blood tests revealed white blood cell (WBC) count was $18.1 \times 10^{9} / \mathrm{L}$ and neutrophilic granulocyte percentage was $89.9 \%$, urine tests showed red and white cells under microscope. Lactate dehydrogenase was 830U/L. After antiinflammation therapy with Cefoperazone sodium and Sulbactam sodium, the patient remained paroxysmal abdominal pain. Nine days after ICD implantation, the enhanced abdominal computed tomography (CT) scan showed a filling defect of the contrast agent in mid-distal of the right renal artery and a cuniform low-density region in the right renal (Fig. 1). Doppler ultrasound showed decreased blood flow in the right renal artery compared with the left side. Hereto, a diagnosis of unilateral acute renal infarction was made. As the patient had no other identified cause for thromboembolism except AF, her renal infarction and thromboembolism of the right renal artery were most likely associated with AF. The patient received anticoagulation immediately, which was enoxaparin $40 \mathrm{mg}$ subcutaneous injection twice a day and warfarin for $2.5 \mathrm{mg}$ Qd after 3 days of enoxaparin, enoxaparin was ceased when the international normalized ratio(INR) reached 2.0 and warfarin was continued. On the 14th after implantation, the abdominal pain was not complained and temperature was normal, blood routine revealed white blood cell count was $8.7 \times 10^{9} / \mathrm{L}$, and neutrophilic granulocyte percentage was $67.4 \%$. After 3-year follow-up, the right renal size reduced from $9.6 \times 4.5 \mathrm{~cm}$ to $4.8 \times 2.2 \mathrm{~cm}$ and serum creatinine increased from $1.13 \mathrm{mg} / \mathrm{dl}$ to $2.38 \mathrm{mg} / \mathrm{dl}$ with a stable INR between 2.0 to 2.5 .

\section{Discussion}

The two leading causes of renal infarction are thromboembolism and in situ thrombosis. The former is an obstruction of a blood vessel by a blood clot from the heart, among which atrial fibrillation is the most common cause ${ }^{[5,6]}$. It is reported that the incidence rate of renal arterial thromboembolism is about $2 \%$ in a series of almost 29862 patients(14 917 men and 14945 women, 50 to 89 years old) with AF following for up to 13 years $^{[7]}$, and other series reported of the prevalence of acute renal infarction(ARI) in paitents from emergency department was $0.013 \%$ and $70 \%$ of the patients had atrial fibrillation in a single center in Japan ${ }^{[8]}$. While a multicentric survey in Turkey reported in 121 renal infarction patients, only 36(about $30 \%$ ) patients had thromboemboli due to atrial fibrillation ${ }^{[9]}$. It seems the incident of ARI varies depending on the characteristics of the cohort (age, gender, comorbidities etc) and the length of follow-up. 
Patients with acute renal infarction usually complain of an acute attack of osphyalgia or diffuse abdominal pain, often accompanied by nausea, vomit, and occasionally fever. The majority of patients may have hematuresis and slightly elevated WBC and C-reactive protein, as well as elevated lactate dehydrogenase levels ${ }^{[4]}$. Because the symptoms of renal infarction are not specific, it is often not diagnosed immediately after the onset, usually more than two days ${ }^{[3,10]}$.

For a patient with osphyalgia, a general CT scan is a preferred initial test, and inflammation, urolithiasis, and other common acute abdominal diseases should be ruled out prior to the diagnosis of renal infarction, because renal infarction is a relatively rare disease in a paitent with osphyalgia or celialgia. On this occasion, if physical examination, $\mathrm{CBC}$, laboratory biochemical analysis, urinalysis, and abdominal Bultrasound could not confirm the cause of abdominal pain, contrast-enhanced CT examination is essential for the diagnosis ${ }^{[4]}$.

In a Turkey survey, the patients enrolled was mainly of middle age, and the final mean creatinine was found to be $1.69 \pm 0.16 \mathrm{mg} / \mathrm{dl}^{[9]}$. As for this elderly patient, her creatinine increased significantly up to $2.38 \mathrm{mg} / \mathrm{dl}$ during 3 years' follow-up. This difference of final serum creatinine may be related to the severity of infarction and the different compensatory ability of the unilateral normal kidney between elderly and middle-aged people. It was reported that acute renal infarction causes irreversible loss of renal mass, but it rarely leads to end-stage renal disease or long-term mortality [11].

In most series studies of thromboembolic renal infarction, the majority of patients received anticoagulation therapy, in which many patients had other anticoagulation indications like $\mathrm{AF}^{[3,12,13]}$. Intravenous heparin or subcutaneous injection of low-molecular-weight heparin(LMWH) followed by oral warfarin is a common approach. This patient received LMWH followed by oral warfarin and maintained INR between 2.0 to 2.5 , which is a standard therapy for renal infarction.

Beyond the diagnosis of renal infarction, the most important problem is what can we learn from this case? If the renal infarction can be avoided or prevented? Is there a better choice of treatment for this patient before thromboembolism happened?

According to ACCP guidelines, the recommendation ${ }^{[14]}$ of antithrombotic therapy for atrial fibrillation for patients with $A F$, including those with paroxysmal $A F$, who are at high risk of stroke (eg, CHADS2 score = 2), oral anticoagulation rather than no therapy was recommended in Grade $1 \mathrm{~A}$ class. This patient had a score of 3 (age $\geq 75$, history of hypertension, and congestive heart failure). The $\mathrm{CHA}_{2} \mathrm{DS}_{2}$-VASc score also estimates the risk of thromboembolism in patients with atrial fibrillation ${ }^{[15]}$. Patients with a $\mathrm{CHA}_{2} \mathrm{DS}_{2}{ }^{-}$ VASc score of 4 to 5 are considered moderate risk[5-10\% annual risk of venous thrombus embolism(VTE)]. And for this patient, congestive heart failure $(+1)$, hypertension $(+1)$, and age $\geq 75$ years $(+2)$, female $(+1)$, made a score of 5 in total. CHADS2 and $\mathrm{CHA}_{2} \mathrm{DS}_{2}-\mathrm{VASc}$ scores both suggested that this patient had a moderate to high risk of thromboembolism, which means anticoagulation therapy should be given when AF first onset, even considering perioperation of ICD. At the mean time, bleeding risk should also be estimated when antithrombolic therapy should be given. The HAS-BLED score is used 
to predict the risk of bleeding for anticoagulation therapy in patients with non-valvular atrial fibrillation ${ }^{[16 \text {, }}$ 17]. This patient had a score of 2 , meaning she was not of high bleeding risk.

As for anticoagulation strategies during cardiac rhythm device periprocedure, it is recommended for unfractionated heparin(UFH) or LMWH as a bridging therapy by American College of Chest Physicians(ACCP) 9th Edition guidelines ${ }^{[14]}$ and American College of Surgeons' Guidelines ${ }^{[18]}$. However, there are some different results published these years. BRUISE CONTROL study is the largest randomized controlled trial to evaluate antithrombotic strategies in patients with Cardiac implantable electronic device(CIED) implantation ${ }^{[16]}$, it showed in 681 patients with an annual risk of thromboembolic events of $5 \%$ or more, compared with the heparin bridging strategy(HBS), continued warfarin treatment during CIED surgery markedly reduced the incidence of clinically significant device-pocket hematoma.

There are several meta-analysis published focusing on the evaluation of bleeding and thromboembolic rate of vitamin $\mathrm{K}$ antagonist and heparin bridging therapy in patients receiving cardiac rhythm devices. In a meta-analysis involving thirteen articles and 5978 patients, the results demonstrated that compared with holding or continuing anticoagulant (AC), HBS significantly increased bleeding events ${ }^{[19]}$. In another review which included 34 studies with 1 randomized trial, the meta-analysis showed there was no difference in the risk of thromboembolic events compared with the bridged and nonbridged groups, and patients receiving perioperational HBS appeared to have an increased risk of overall and major bleeding, and a similar risk of thromboembolic events compared with non-HBS patients ${ }^{[20]}$. Ghanbari et al. identified 8 studies enrolling 2,321 patients in a meta-analysis ${ }^{[21]}$, compared with heparin-bridging therapy, a strategy of continuous warfarin therapy throughout CIEDs implantation is associated with a decreased risk of bleeding without increased risk of thromboembolic events. In another meta-analysis evaluating patients on chronic oral anticoagulant (OAC) therapy undergoing CIEDs implantation, it is indicated that implantation of CIEDs with uninterrupted OAC therapy seemed better than both of HBS and OAC interruption therapy for lowering the bleeding risk ${ }^{[22]}$. In a comparation of continuous oral anticoagulant vesus HBS in patients undergoing CIED surgery, seven studies with totaling 2,191 patients were included in a meta-analysis, continous OAC was associated with a significantly lower risk of postprecedure bleeding risk compared with HBS, and there was no difference in the thromboembolic events between two strategies ${ }^{[23]}$.

Network meta-analysis (NMA) has some advantages over traditional meta-analysis. A network metaanalysis of observational studies tested the antithrombotic therapy during the CIED implantation, in which thirty-one observational studies, 41,174 patients were included. Continued warfarin, interrupted warfarin, and interrupted NOACs all reduced the risk of bleeding compared with HBS, and interrupted NOACs therapy might be the optimal anticoagulation strategy for the perioperative period of CIED implantation ${ }^{[24]}$.

As can be seen from the above random controlled study and meta-analysis, continuous oral anticoagulation does not increase the bleeding risk and may reduce thromboembolic events, it may be 
the best option for perioperative CIED implantation. Some authors suggested HBS was given only in patients at high risk of stroke when anticoagulation was interrupted.

\section{Conclusion}

For this elderly female patient with moderate to high risk of thromboembolism and low bleeding risk, appropriate anticoagulation like OAC can prevent thromboembolic events due to paroxysmal atrial fibrillation. If anticoagulation was given before ICD implantation, renal infarction might be be avoided, and according to evidence-based medical study results, there was no need to interrupt OAC during ICD implantation if she had received anticoagulant before.

In medical practice, anticoagulations should be given to patients with atrial fibrillation according to guideline's recommendation to avoid thromboembolism events if there is no contraindication or any other unapplicable conditions.

\section{Declarations}

- 1.Ethical Approval and Consent to participate: This case report has been approved by the Biomedical Ethics Committee, Beijing Friendship Hospital, Capital Medical University, the consent to participate is not applicable.

- 2. Consent for publication: All the authors agreed with publication.

- 3.Availability of data and materials: The data and materials is available.

- 4.Competing interests: There is no conflict of interest.

- 5.Funding: No Funding/ Not applicable.

- 6.Authors' contributions: Yingshuo Huang collected data and wrote paper, Ying Sun revised and reviewed the paper.

- 7.Acknowledgements: None/Not applicable.

- 8. Authors' information:

Ying-shuo Huang, ${ }^{1}$ Ying Sun. ${ }^{2}$

1 Department of the Research Ward, Beijing Friendship Hospital, Capital Medical University, Beijing, 100050, China.

2 Department of Geriatrics, Beijing Friendship Hospital, Capital Medical University, Beijing, 100050, China.

Corresponding authors: Dr. Ying Sun

Department of Geriatrics, Beijing Friendship Hospital, Capital Medical University, No.95 Yong'An Road, XiCheng District, Beijing, 100050, China, 
Email: yingsun15@163.com Phone: +86 (0)10 63139047

\section{References}

1. Charlier C, Perrodeau E, Leclercq A, et al. Clinical features and prognostic factors of listeriosis: the MONALISA national prospective cohort study. The Lancet Infectious diseases. 2017;17(5):510-9. DOI 10.1016/S1473-3099(16)30521-7.

2. Bourgault $M$, Grimbert $P$, Verret $C$, et al. Acute renal infarction: a case series. Clinical journal of the American Society of Nephrology: CJASN. 2013;8(3):392-8. DOI 10.2215/CJN.05570612.

3. Hazanov N, Somin M, Attali M, et al. Acute renal embolism. Forty-four cases of renal infarction in patients with atrial fibrillation. Medicine. 2004;83(5):292-9. DOI

10.1097/01.md.0000141097.08000.99.

4. Antopolsky M, Simanovsky N, Stalnikowicz R, et al. Renal infarction in the ED: 10-year experience and review of the literature. The American Jjournal of Emergency Medicine. 2012;30(7):1055-60. DOI 10.1016/j.ajem.2011.06.041.

5. Geller BJ, Giugliano RP, Braunwald E, et al. Systemic, noncerebral, arterial embolism in 21,105 patients with atrial fibrillation randomized to edoxaban or warfarin: results from the Effective Anticoagulation With Factor Xa Next Generation in Atrial Fibrillation-Thrombolysis in Myocardial Infarction Study 48 trial. American HeartJournal. 2015;170(4):669-74. DOI 10.1016/j.ahj.2015.06.020.

6. Komolafe B, Dishmon D, Sultan W, et al. Successful aspiration and rheolytic thrombectomy of a renal artery infarct and review of the current literature. The Canadian journal of cardiology. 2012;28(6):760 e761-763.DOI 10.1016/j.cjca.2012.06.020.

7. Frost L, Engholm G, Johnsen S, et al. Incident thromboembolism in the aorta and the renal, mesenteric, pelvic, and extremity arteries after discharge from the hospital with a diagnosis of atrial fibrillation. Arch Intern Med. 2001;161(2):272-6. DOI 10.1001/archinte.161.2.272.

8. Nagasawa T, Matsuda $K$, Takeuchi $Y$, et al. A case series of acute renal infarction at a single center in Japan. Clin Exp Nephrol. 2016;20(3):411-5. DOI 10.1007/s10157-015-1168-1.

9. Eren N, Gungor O, Kocyigit I, et al. Acute renal infarction in Turkey: a review of 121 cases. Int Urol Nephrol. 2018;50(11):2067-72. DOI 10.1007/s11255-018-1979-6.

10. Tsai SH, Chu SJ, Chen SJ, et al. Acute renal infarction: a 10-year experience. Int J Clin Pract. 2007;61(1):62-7. DOI 10.1111/j.1742-1241.2006.01136.x.

11. Yun WS. Long-term follow-up results of acute renal embolism after anticoagulation therapy. Ann Vasc Surg. 2015;29(3):491-5. DOI 10.1016/j.avsg.2014.09.028.

12. Bolderman R, Oyen R, Verrijcken $A$, et al. Idiopathic renal infarction. The American Journal of Medicine. 2006;119(4):356 e359-312.DOI 10.1016/j.amjmed.2005.06.049.

13. Korzets Z, Plotkin E, Bernheim J, et al. The clinical spectrum of acute renal infarction. The Israel Medical Association journal: IMAJ. 2002;4(10):781-4. 
14. You JJ, Singer DE, Howard PA, et al. Antithrombotic therapy for atrial fibrillation: Antithrombotic Therapy and Prevention of Thrombosis, 9th ed: American College of Chest Physicians EvidenceBased Clinical Practice Guidelines. Chest. 2012;141(2 Suppl):e531S-575S. DOI 10.1378/chest.112304.

15. Friberg L, Rosenqvist M, Lip GY. Evaluation of risk stratification schemes for ischaemic stroke and bleeding in 182678 patients with atrial fibrillation: the Swedish Atrial Fibrillation cohort study. Eur Heart J. 2012;33(12):1500-10. DOI 10.1093/eurheartj/ehr488.

16. Birnie DH, Healey JS, Wells GA, et al. Pacemaker or defibrillator surgery without interruption of anticoagulation. N Engl J Med. 2013;368(22):2084-93. DOI 10.1056/NEJMoa1302946.

17. Roldan V, Marin F, Manzano-Fernandez S, et al. The HAS-BLED score has better prediction accuracy for major bleeding than CHADS2 or CHA2DS2-VASc scores in anticoagulated patients with atrial fibrillation. J Am Coll Cardiol. 2013;62(23):2199-204. DOI 10.1016/j.jacc.2013.08.1623.

18. Hornor MA, Duane TM, Ehlers AP, et al. American College of Surgeons' Guidelines for the Perioperative Management of Antithrombotic Medication. J Am Coll Surg. 2018;227(5):521-36 e521. DOI 10.1016/j.jamcollsurg.2018.08.183.

19. Bernard ML, Shotwell M, Nietert PJ, et al. Meta-analysis of bleeding complications associated with cardiac rhythm device implantation. Circulation Arrhythmia electrophysiology. 2012;5(3):468-74. DOI 10.1161/CIRCEP.111.969105.

20. Siegal D, Yudin J, Kaatz S, et al. Periprocedural heparin bridging in patients receiving vitamin $\mathrm{K}$ antagonists: systematic review and meta-analysis of bleeding and thromboembolic rates. Circulation. 2012;126(13):1630-9. DOI 10.1161/CIRCULATIONAHA.112.105221.

21. Ghanbari H, Phard WS, Al-Ameri $\mathrm{H}$, et al. Meta-analysis of safety and efficacy of uninterrupted warfarin compared to heparin-based bridging therapy during implantation of cardiac rhythm devices. The American journal of cardiology. 2012;110(10):1482-8. DOI 10.1016/j.amjcard.2012.06.057.

22. Du L, Zhang $Y$, Wang $W$, et al. Perioperative anticoagulation management in patients on chronic oral anticoagulant therapy undergoing cardiac devices implantation: a meta-analysis. Pacing clinical electrophysiology: PACE. 2014;37(11):1573-86. DOI 10.1111/pace.12517.

23. Sant'anna RT, Leiria TL, Nascimento T, et al. Meta-analysis of continuous oral anticoagulants versus heparin bridging in patients undergoing CIED surgery: reappraisal after the BRUISE study. Pacing clinical electrophysiology: PACE. 2015;38(4):417-23. DOI 10.1111/pace.12557.

24. $\mathrm{He} \mathrm{H}, \mathrm{Ke} \mathrm{BB}, \mathrm{Li} \mathrm{Y}$, et al. Perioperative management of antithrombotic therapy in patients receiving cardiovascular implantable electronic devices: a network meta-analysis. Journal of Interventional Cardiac Electrophysiology: an international journal of arrhythmias pacing. 2017;50(1):65-83. DOI 10.1007/s10840-017-0280-4.

\section{Figures}


A

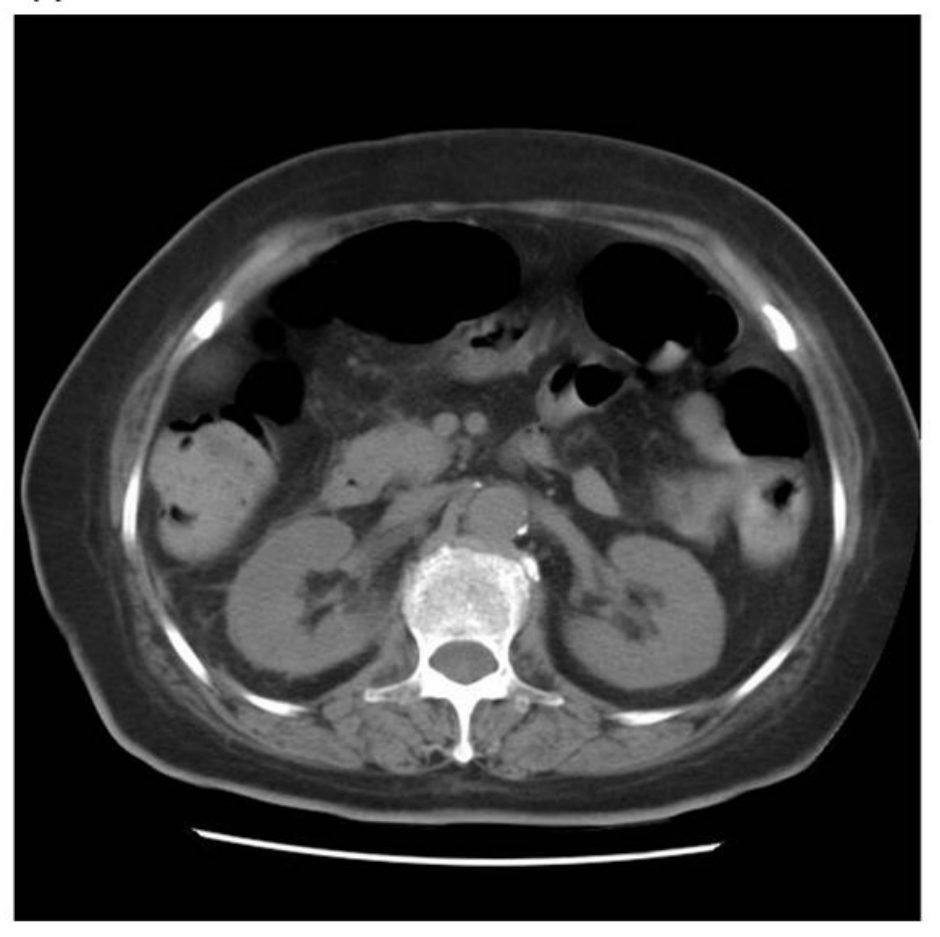

\section{B}

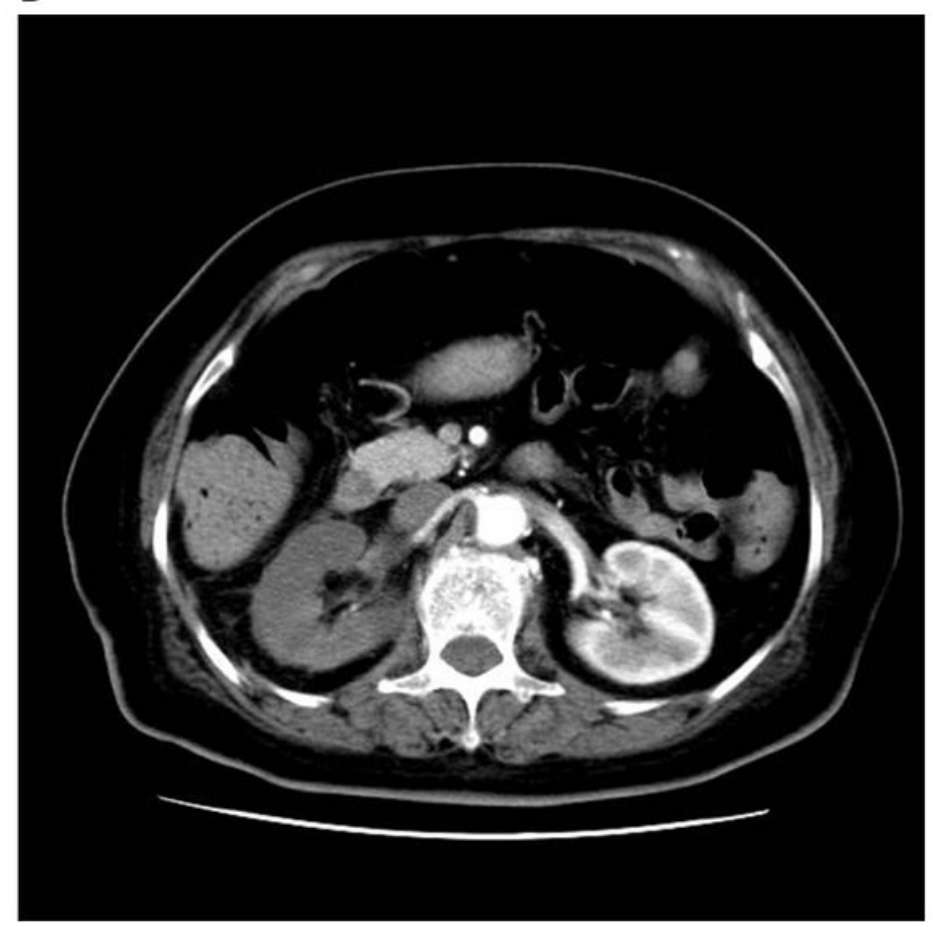

Figure 1

General and contrast-enhanced abdominal CT scan A. Abdominal general CT scan, both kidney can be seen with similar density; B. Abdominal contrast-enhanced CT scan, the right kidney artery and right 